\title{
Developing a new PET myocardial perfusion tracer
}

\author{
Diwakar Jain, MD, FACC, FASNC, ${ }^{a}$ Afshin Ghanbarinia, MD, ${ }^{\mathrm{a}}$ \\ and Zuo-Xiang He, $M^{b}$
}

\section{See related article, pp. 763-768}

Myocardial perfusion imaging is the most commonly used procedure in nuclear medicine. ${ }^{1}$ Availability of thallium-201 made it possible to study myocardial perfusion non-invasively at rest and with exercise in mid70s. ${ }^{2}$ Development of Tc-99m based perfusion tracers ( ${ }^{99 \mathrm{~m}}$ Tc-sestamibi and ${ }^{99 \mathrm{~m}}$ Tc-ttrofosminin) in early $90 \mathrm{~s}$ overcame many of the limitations of Tl-201 and is largely responsible for MPI becoming the centerpiece of non-invasive techniques for the evaluation of coronary artery disease. ${ }^{3}$ Despite the widespread use of ${ }^{99 \mathrm{~m}} \mathrm{Tc}$ labeled myocardial perfusion tracers, artifacts due to attenuation and extra-cardiac activity continue to be important limitations of these agents. Furthermore, these agents do not provide quantitative information about myocardial blood flow. Therefore, the search for an ideal perfusion tracer is still on. Positron emission tomography (PET) offers several advantages over single photon emission computed tomography (SPECT): better imaging characteristics due to higher photon flux, more robust attenuation correction, shorter image acquisition time and possibility of obtaining quantitative information about myocardial perfusion. It is thought PET imaging may be able to overcome the limitations of currently used SPECT perfusion tracers. Whereas a number of PET tracers are currently available for myocardial perfusion imaging, they all suffer from significant limitations:

From the Section of Cardiovascular Medicine and Cardiovascular Nuclear Imaging Laboratory, ${ }^{a}$ Drexel University College of Medicine, Philadelphia, PA; Department of Nuclear Medicine, ${ }^{\mathrm{b}}$ Fu Wai Hospital, Chinese Academy of Medical Sciences, Beijing, China.

Reprint requests: Diwakar Jain, MD, FACC, FASNC, Section of Cardiovascular Medicine and Cardiovascular Nuclear Imaging Laboratory, Drexel University College of Medicine, MS 470, 245 N. 15th Street, Philadelphia, PA 19102; Diwakar.Jain@Drexelmed.edu. J Nucl Cardiol 2009;16:689-90.

$1071-3581 / \$ 34.00$

Copyright $(2009$ by the American Society of Nuclear Cardiology. doi:10.1007/s12350-009-9113-x
${ }^{13} \mathrm{~N}$-ammonia and ${ }^{15} \mathrm{O}$-water require on-site cyclotron for their production, ${ }^{82} \mathrm{Rb}$ is generator produced, but given an extremely short half-life this can only be used with pharmacological stress. Therefore, attention is turning toward developing ligands, which can be labeled with generator produced PET radionuclides, or with cyclotron-produced radionuclides with relatively longer halflife, which do not necessarily require on-site cyclotrons. In this respect, gallium-68 and copper-64 are potentially attractive PET tracers, which can be eluted from generators with long shelf life. However, so far no suitable ligands have been developed to exploit the use of ${ }^{68} \mathrm{Ga}$ and ${ }^{64} \mathrm{Cu}$ as myocardial perfusion tracers. Interestingly, $\mathrm{Yu}$ and colleagues have presented preliminary experimental data in this issue of the journal on BMS-74715802 , a pyridazinone analog, which can be labeled with F-18 and used as a myocardial perfusion tracer. ${ }^{4}$

Any new potential myocardial perfusion imaging agent has to undergo a series of elaborate studies to characterize its biokinetics and behavior in in vitro and in vivo studies to detect any possible interaction with a multitude of physiological and pharmacological variables. Myocardial uptake of an ideal myocardial perfusion tracer should correlate directly and linearly with myocardial blood flow over a wide range and should not be affected by other metabolic and physiological variables.

$\mathrm{Yu}$ and colleagues studied myocardial and other organ uptake of ${ }^{18} \mathrm{~F}$-BMS747158-02 under fasting and non-fasting conditions and with the use of two different anesthetic agents (pentobarbital and ketamine/xylazine) to determine any effects of fed/fasting status or the choice of anesthetic agent on myocardial tracer uptake. ${ }^{4}$ As expected none of these conditions had any effect on myocardial tracer uptake, which remained approximately in the range of $3 \% \mathrm{ID} / \mathrm{g}$. The tracer uptake by other organs was relatively low compared to the myocardial uptake and largely remained unchanged under different physiological conditions. They compared this with myocardial and other organ uptake of ${ }^{18} \mathrm{~F}-\mathrm{FDG}$. Interestingly, they found up to 14-fold variation in myocardial ${ }^{18}$ F-FDG uptake between fed and fasting conditions and between the two anesthetics used in this study (pentobarbital and ketamine/xylazine). The latter is known to interfere with insulin release from the 
pancreas, which results in an increase in blood sugar, but a decrease in myocardial glucose uptake. Myocardial uptake of ${ }^{18} \mathrm{~F}$-FDG was 14 -fold less under prolonged fasting conditions compared to fed conditions and the myocardium could not be visualized well on images acquired 15 and 60 minutes post ${ }^{18}$ F-FDG injection.

The results of this study are also relevant for the use of ${ }^{18} \mathrm{~F}$-FDG for the detection of exercise-induced myocardial ischemia. Our group has recently shown that ${ }^{18}$ F-FDG injected at peak exercise under fasting conditions is taken up selectively by the ischemic myocardium and this can be used as a hot spot imaging agent for the detection of CAD in clinical practice. ${ }^{5-8}$ Under fasting conditions (particularly after fasting for over 12 hours), normal myocardium has very little myocardial ${ }^{18} \mathrm{~F}-\mathrm{FDG}$ uptake due to low blood sugar and insulin levels. However, myocardial ischemia results in a profound increase in regional glucose and ${ }^{18} \mathrm{~F}-\mathrm{FDG}$ uptake, which is not dependent upon blood glucose and insulin levels. This results in a significant differential in ${ }^{18}$ F-FDG uptake between normally perfused and ischemic myocardium. $\mathrm{Yu}$ et al found a 14-fold reduction in myocardial ${ }^{18}$ F-FDG uptake in rats fasted for 18 hours compared to fed rats. This is consistent with our clinical studies, where ${ }^{18}$ F-FDG uptake following its injection at peak exercise in patients with coronary artery disease fasted for $\geq 12$ hours was seen only in ischemic myocardium. ${ }^{6-8}$

$\mathrm{Yu}$ et al studied only resting myocardial uptake of ${ }^{18}$ F-BMS-747158-02 and ${ }^{18} \mathrm{~F}$-FDG in normal rats under different physiological studies. It would have been very helpful had the authors also included a parallel group of animals, who were given a catecholamine agent under fasting or fed conditions just before the administration of ${ }^{18}$ F-BMS747158-02 or ${ }^{18}$ F-FDG to mimic the effect of exercise to determine the differences between ${ }^{18} \mathrm{~F}-\mathrm{BMS} 747158-02$ and ${ }^{18} \mathrm{~F}-\mathrm{FDG}$ myocardial uptake at rest and on pharmacological stress. It would be very interesting to see a comparison between myocardial uptake of ${ }^{18}$ F-BMS747158-02 and ${ }^{18}$ F-FDG at rest and with stress in animal models with inducible ischemia and in patients with coronary artery disease in future studies. In the clinical studies by $\mathrm{He}$ et al, exercise ${ }^{18} \mathrm{~F}$-FDG showed greater extent of myocardial ischemia compared to reversible perfusion abnormalities on exercise-rest ${ }^{99 \mathrm{~m}} \mathrm{Tc}$-sestamibi imaging. ${ }^{6-8}$ Whether exercise-rest ${ }^{18} \mathrm{~F}$-BMS747158-02 would provide information closer to that with exercise ${ }^{18}$ F-FDG than exercise-rest SPECT perfusion imaging is difficult to speculate at this stage. Nevertheless, ${ }^{18}$ F-BMS747158-02 appears to be a very promising new myocardial perfusion tracer, which warrants further clinical evaluation.

\section{References}

1. Jain D, Zaret BL. Nuclear imaging in cardiovascular medicine. In: Rosendorf C, editor. Essentials of cardiovascular medicine. 2nd ed. Totowa, NJ: Humana Press; 2005. p. 221-44.

2. Wackers FJ, Sokole EB, Samson G, Schoot JB, Lie KI, Liem KL, et al. Value and limitations of thallium-201 scintigraphy in the acute phase of myocardial infarction. N Engl J Med 1976;295:1-5.

3. Jain D. ${ }^{99 \mathrm{~m}}$ Technetium labeled myocardial perfusion imaging agents. Semi Nucl Med 1999;29:221-36.

4. Yu Ming, Guarldi M, Bozek J, Kagan M, Azure M, Rdeke H, et al. Effects of food intake and anesthetic on cardiac imaging and uptake of BMS747158-02 in comparison with FDG. J Nucl Cardiol 2009 (current issue).

5. Jain D, McNulty PH. Exercise-induced myocardial ischemia: Can this be imaged with F-18-fluorodeoxyglucose? J Nucl Cardiol 2000;7:286-8 (editorial).

6. He ZX, Shi RF, Wu YJ, Tian YQ, Liu XJ, Wang SW, et al. Direct imaging of exercise induced myocardial ischemia in coronary artery disease. Circulation 2003;108:1208-13.

7. Jain D, He ZX. Direct imaging of myocardial ischemia: A potential new paradigm in nuclear cardiovascular imaging. J Nucl Cardiol 2008; 15:617-30.

8. Dou KF, Yang MF, Yang YJ, Jain D, He ZX. Myocardial ${ }^{18}$ FDG uptake after exercise-induced myocardial ischemia in patients with coronary artery disease. J Nucl Med 2008;49:1986-91. 